\title{
Tunable plasmonic glucose sensing based on the dissociation of Con A-aggregated dextran-coated gold colloids
}

\author{
Kadir Aslan ${ }^{\text {a }}$, Joseph R. Lakowicz ${ }^{\mathrm{b}, 1}$, Chris D. Geddes ${ }^{\mathrm{a}, \mathrm{b}, *}$

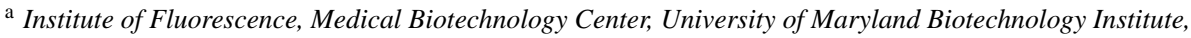 \\ 725 West Lombard Street, Baltimore, MD 21201, USA \\ ${ }^{\mathrm{b}}$ Center for Fluorescence Spectroscopy, Department of Biochemistry and Molecular Biology, Medical Biotechnology Center, \\ University of Maryland School of Medicine, 725 West Lombard Street, Baltimore, MD 21201, USA
}

Received 14 October 2003; received in revised form 16 April 2004; accepted 28 April 2004

\begin{abstract}
We describe a new approach for glucose determination with tunable glucose dynamic sensing ranges, dependent on the properties of new nanosensors, which are comprised of Con A-aggregated dextran-coated gold colloids.

Dextran-coated 10 or $20 \mathrm{~nm}$ gold colloids can be aggregated with Con A in a controlled fashion, the change in absorbance at an arbitrary wavelength used to monitor the extent of aggregation, which can be optimized for sensing. The presence of any glucose competitively binds with Con A, dissociating the dextran-coated colloids, affording for the reverse gold plasmon change and hence the determination of glucose concentrations. For one of our sensor systems, a $500 \mathrm{~K}$ dextran-20 nm gold sensor, crosslinked with $8.70 \mu \mathrm{M}$ Con A, a change in absorbance at $650 \mathrm{~nm}$ of 0.03 was observed, in response to as little as $3 \mathrm{mM}$ glucose. In contrast, a 500K dextran-10 nm gold $18.7 \mu \mathrm{M}$ Con A aggregate sensor, produced a 0.05 and 0.1 change in absorbance, respectively, by the addition of 50 and $100 \mathrm{mM}$ glucose.

We have found that the glucose sensing ranges can be somewhat tuned by altering the properties, and therefore the extent of aggregation of the gold aggregate sensors. Reducing the gold colloid size and dextran molecular weight typically reduces the glucose sensing range (lower [glucose]) but also reduces the long-term stability of the gold aggregate sensor. Similarly the concentration of Con A used to aggregate the system also has an effect on long term sensor stability and glucose response.

In this paper, we present our findings, which offer unique opportunities and perspectives for building tunable plasmonic type glucose sensors.
\end{abstract}

(C) 2004 Elsevier B.V. All rights reserved.

Keywords: Gold colloids; Tunable glucose sensor(s); Plasmonic nanosensors

\section{Introduction}

Diabetes results in long-term health disorders including cardiovascular disease and blindness. One of the major challenges in the management of diabetes is the monitoring of glucose concentrations. Despite intensive efforts [1-5], no method is currently available for the continuous non-invasive monitoring of blood glucose.

Metal nanostructures have been studied extensively and are emerging as important colorimetric reporters due to their high extinction coefficients, which are typically several

\footnotetext{
* Corresponding author.

E-mail address: geddes@umbi.umd.edu (C.D. Geddes).

${ }^{1}$ Co-corresponding author.
}

orders of magnitude larger than those of organic dyes [6]. In particular, nanostructures made from the noble metals, such as those of silver or gold, with their associated strong plasmon resonance, have generated great interest [6-13]. Gold nanoparticles display plasmon absorption bands that depend on their size and shape [7-9]. Nanoparticle aggregation results in further color changes of gold nanoparticle solutions due to mutually induced dipoles that depend on interparticle distance and aggregate size [10-13]. The fact that the plasmon resonance is a sensitive function of nanostructure geometry, coupled with synthetic advances that allow for the controlled and systematic variations in nanostructure geometry, is leading to the expanse of this sensing field called "plasmonics". Gold nanoparticle aggregation induced by analytes has been demonstrated for DNA 

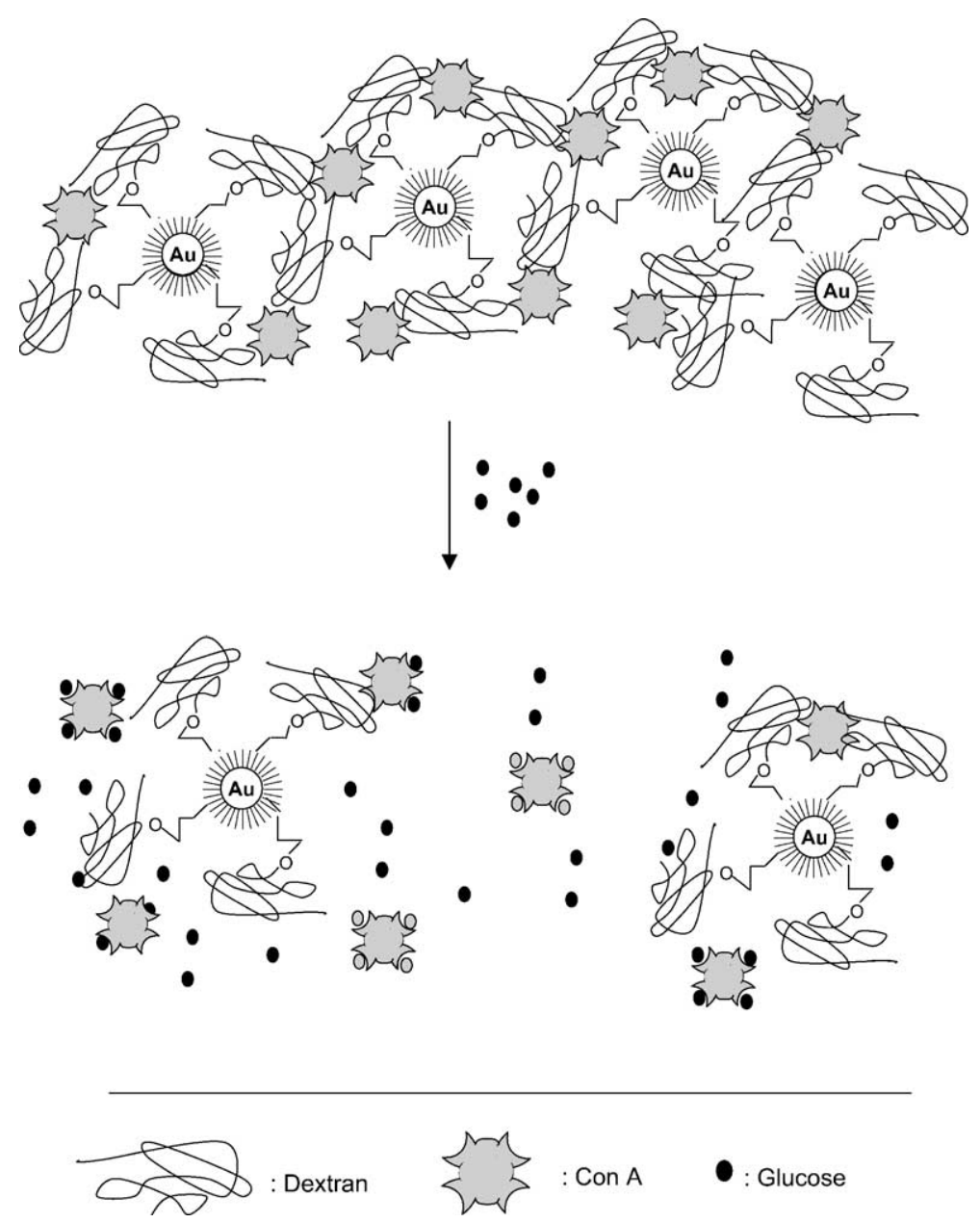

Fig. 1. Glucose sensing mechanism. The dissociation of Con A-aggregated dextran-coated gold colloids.

$[14,15]$, metal ions [16,17] and antibodies [18]. However, to the best of our knowledge, their use in glucose sensing has been ill-explored.

In this paper, we report how dextran-coated gold colloids, which have been aggregated by the controlled addition of Con A, provide for useful sensing aggregates, which show plasmon changes in the presence of glucose which is widely known to competitively bind Con A (Fig. 1). Further, by altering the gold colloid size, the dextran molecular weight and the concentration of Con A used to form the sensing aggregate, we can to some degree tune the dynamic glucose sensing range.

\section{Experimental}

\subsection{Materials}

Gold nanoparticle dispersions (monodisperse, either 20 or $10 \mathrm{~nm}$ average particle diameter), concanavalin A (Con A from Canavalia ensiformis), dextran (average molecular weight: 64000 and 505000 ) hydrogen peroxide, sulfuric acid, sodium phosphate monobasic, phosphate-buffered saline (PBS), absolute ethanol, 2-(2-aminoethoxy)ethanol (AEE) and $N$-hydroxy-2,5-pyrrolidinedione (NHS) were obtained from Sigma. 16-Mercaptohexadecanoic acid (16-MHDA) and polyoxyethylene (20) sorbitan monolaurate (Tween 20), epichlorohydrin, 2-methoxyethyl ether (diglyme), and nitric acid were obtained from Aldrich. $N$-3-(Dimethylaminopropyl)- $N N^{\prime}$-ethyl-carbodiimide (EDC) was obtained from Fluka. All chemicals were used as received.

\subsection{Buffers and solutions}

Sodium phosphate monobasic buffer solution was prepared to a $10 \mathrm{mM}$ concentration at $\mathrm{pH}$ 7. PBS was dissolved in deionized water and the $\mathrm{pH}$ was adjusted to 7.4. Exact $\mathrm{pH}$ values for buffer solutions were obtained using a Beckman $\mathrm{pH}$ meter. Deionized water $(>18 \mathrm{M} \Omega / \mathrm{cm})$ was used in the preparation of all buffer solutions. All glassware was washed with "piranha solution" $\left(3: 7,30 \% \mathrm{H}_{2} \mathrm{O}_{2} / \mathrm{H}_{2} \mathrm{SO}_{4}\right)$ prior to use.

Solutions of $0.50 \mathrm{mM}$ 16-MHDA were prepared in degassed ethanol. Tween 20 solutions were prepared in sodium phosphate buffer at $\mathrm{pH} 7$. 


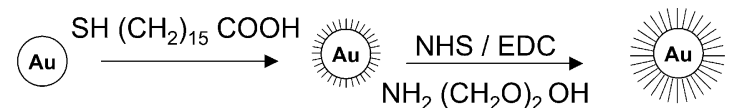

(A) Unmodified

Au nanoparticles $(10 / 20 \mathrm{~nm}$ in diameter $)$

(B) AEE-modified Au nanoparticles

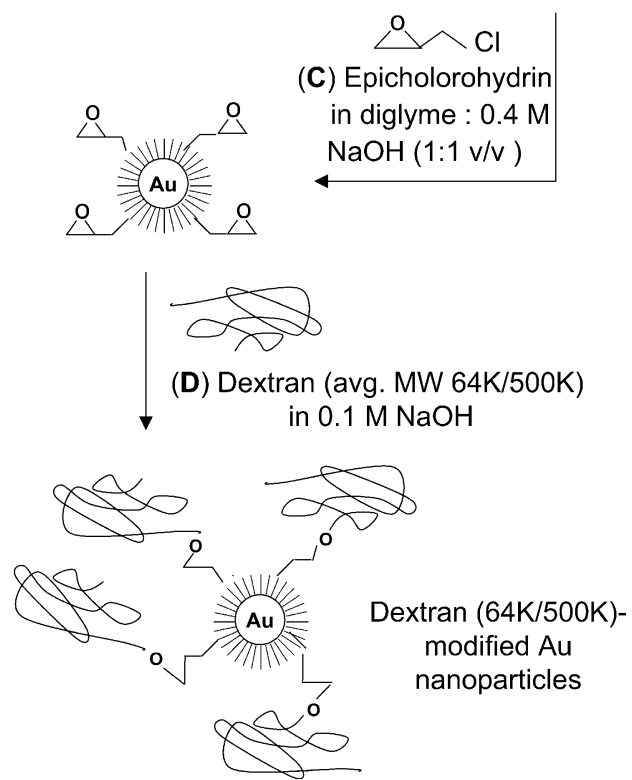

Fig. 2. Synthetic scheme for the preparation of the dextran-coated gold colloids.

\subsection{Surface modification of the gold colloids: preparation of the glucose aggregate nanosensors}

The immobilization of dextran on gold nanoparticles was performed using the following four steps: (A) chemisorption of a long-chain carboxyl-terminated alkane thiol on gold nanoparticles as described previously [19]; (B) the activation of surface carboxyl groups using EDC and NHS; (C) activation of hydroxyl groups using epicholorohydrin; and (D) the covalent coupling of dextran (Fig. 2).

Gold nanoparticle dispersions with a concentration of $0.80 \mathrm{nM}$ for $20 \mathrm{~nm}$, and $8 \mathrm{nM}$ for $10 \mathrm{~nm}$ gold colloids (determined by measuring absorbance at $520 \mathrm{~nm}$ and using extinction coefficients of $1.25 \times 10^{9}$ and $1.21 \times 10^{8} \mathrm{M}^{-1} \mathrm{~cm}^{-1}$ for 20 and $10 \mathrm{~nm}$ gold, respectively; Sigma) were degassed with nitrogen before use. The gold colloids are not naked, but indeed solution stabilized with the citrate counter ion. The addition of the alkane thiol (step 1) replaces the citrate counter ion, producing a stabilized monolayered protected particle [19]. Equal volumes $(400 \mu l)$ of gold nanoparticle dispersions $(0.80 / 8 \mathrm{nM}$, before mixing) and Tween $20(1.82 \mathrm{mg} / \mathrm{ml}$, before mixing) in $\mathrm{pH} 7$ buffer were gently mixed and allowed to stand for $30 \mathrm{~min}$ for the physisorption of the Tween 20 to the gold nanoparticles [19]. Four hundred microliters of $0.50 \mathrm{mM}$ 16-MHDA was then added and the final mixture (final concentrations: [gold nanoparticles] $=0.27 / 2.67 \mathrm{nM}$; [Tween 20] $=0.61 \mathrm{mg} / \mathrm{ml}$; [16-MHDA $]=0.17 \mathrm{mM}$ ) was allowed to stand for $3 \mathrm{~h}$ for the chemisorption of 16-MHDA to be completed on the gold colloids, while simultaneously displacing Tween 20 [19]. In order to remove excess 16-MHDA and Tween 20, the final mixture was centrifuged (three times for $15 \mathrm{~min}$ at $16060 \times \mathrm{g}$; the supernatants were discarded after each cycle) and resuspended in phosphate buffer (with $1.82 \mathrm{mg} / \mathrm{ml}$ Tween 20 at $\mathrm{pH}$ 7). 16-MHDA-modified gold colloids that remained in the centrifugate were then reacted with a mixture of freshly prepared $50 \mathrm{mM}$ NHS and $200 \mathrm{mM}$ EDC solution (in phosphate buffer without Tween 20) for $5 \mathrm{~min}$. The resulting nanoparticle dispersion was centrifuged $(5 \mathrm{~min}, 16060 \times \mathrm{g})$ and after discarding the supernatant, the remaining NHS ester-alkane thiol-modified gold nanoparticles were reacted with a freshly prepared solution of AEE $(2 \%, v / v)$ for $10 \mathrm{~min}$. Excess AEE was removed by centrifugation (for $5 \mathrm{~min}$ at $16060 \times g$ at least three times). The retentate that contained AEE-modified gold nanoparticles was centrifuged $(5 \mathrm{~min}, 16060 \times g)$. The hydroxyl groups on the AEE-modified gold nanoparticles were activated with $0.6 \mathrm{M}$ epicholorohydrin solution in a $1: 1$ mixture of $0.4 \mathrm{M} \mathrm{NaOH}$ and diglyme for $4 \mathrm{~h}$ at room temperature. The nanoparticle dispersion was then centrifuged for $10 \mathrm{~min}$ at $16060 \times \mathrm{g}$ and resuspended in diglyme and centrifuged again to remove the excess epicholorohydrin. The centrifugate, containing AEE-modified gold nanoparticles with active epoxide groups, were incubated in dextran solution $(0.1 \mathrm{M} \mathrm{NaOH})$ for $20 \mathrm{~h} \mathrm{[20].} \mathrm{Finally,} \mathrm{dextran-modified} \mathrm{gold} \mathrm{nanoparticles}$ were centrifuged for $15 \mathrm{~min}$ at $16060 \times g$ and resuspended in $0.1 \mathrm{M} \mathrm{NaOH}$ and centrifuged four more times to remove the excess dextran. All solutions of dextran-coated gold nanoparticles were stored in polypropylene centrifuge tubes in the dark to prevent light-induced flocculation of the nanoparticles and oxidation of the alkane thiols [21].

\subsection{Methods}

All absorption measurements were performed using a Varian UV-vis 50 spectrophotometer using Quartz cuvettes (Starna). Standard Varian kinetic software was used in the data analysis.

\section{Results and discussion}

Our gold plasmonic type glucose sensors are based on the well-known fact that the nanoparticle solutions display changes in their plasmon absorption spectrum upon aggregation and/or surface modification [7-13]. Fig. 3 shows both a shift and broadening of the gold plasmon band at $\approx 520 \mathrm{~nm}$ as the colloids are homogeneously coated with dextran (steps A $\rightarrow$ D, cf. Fig. 2).

To optimize the extent of aggregation, and therefore the dynamic range for dissociation upon glucose addition, we aggregated $500 \mathrm{~K}$ dextran-coated $20 \mathrm{~nm}$ gold colloids (500K, $20 \mathrm{~nm}$ gold), with different concentrations of Con A, which is well known to competitively bind dextran [22,23] (Fig. 4). 


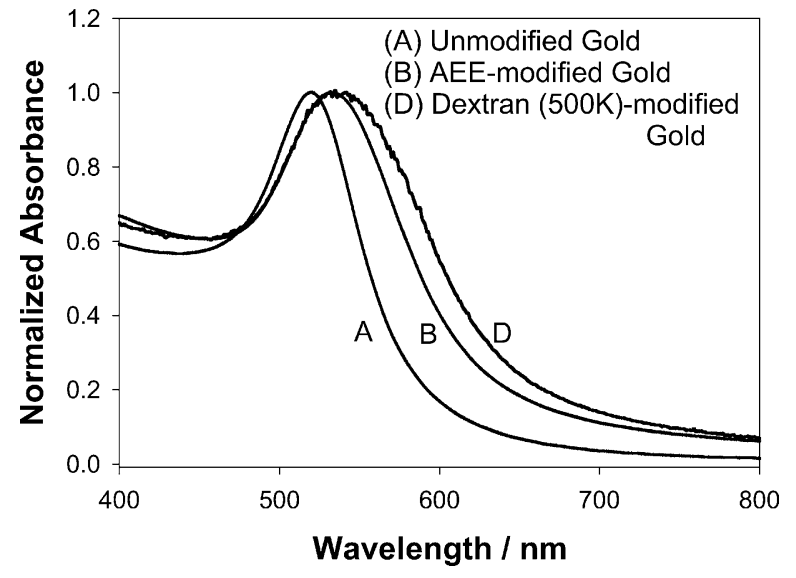

Fig. 3. Normalized absorption spectrum for: (A) unmodified $20 \mathrm{~nm}$ gold colloids; (B) AEE-modified colloids; and (D) dextran-coated $20 \mathrm{~nm}$ gold colloids.

The normalized spectra show both a broadening and a slight red-shift as a function of increased Con A concentration, simply reflecting the close proximity of increasingly aggregated gold colloids and their mutually induced dipole interactions [10-13], a function of the affinity of Con A for dextran. We chose a wavelength of $650 \mathrm{~nm}$ to monitor the extent of aggregation, which clearly showed a significant change in absorbance, $\Delta A_{650}$, between a highly aggregated system (no. 1, Fig. 4, top), and a slightly aggregated system (no. 7). We additionally investigated the time to complete aggregation for the same set of samples by monitoring the $\Delta A_{650}$ as a function of time. As expected, samples with greater additions of Con A showed shorter $90 \%$ absorbance (of the final absorbance maximum value) change times, simply reflecting a quicker aggregation rate. For all subsequent studies, the aggregates were allowed to form well past their $90 \%$ $\Delta A_{650}$ value before use. Indeed, for glucose additions to the nanoaggregate sensors for sensing, $90 \% \Delta A_{650}$ values are quoted due to the equilibrium between dextran-Con $\mathrm{A}$ and glucose.

Fig. 5 shows a range of dextran-coated gold aggregates, to which glucose was added. Fig. 5 (top) shows the response of a $500 \mathrm{~K}$ dextran- $20 \mathrm{~nm}$ gold colloid which has been pre-aggregated past its $90 \% \Delta A_{650}$ value with both 37.3 and $8.7 \mu \mathrm{M}$ Con A, towards glucose. Surprisingly, greater $\Delta A_{650}$ values were observed for the lesser-aggregated system, i.e. less Con A. After studying many concentrations of Con A, we have determined that highly aggregated systems ( $>60 \mu \mathrm{M}$ Con A) can lead to turbid solutions, having sufficiently enough Con A to require evermore glucose to attain aggregate disassociation, reducing the sensing range (higher [glucose]). In contrast, while weakly aggregated

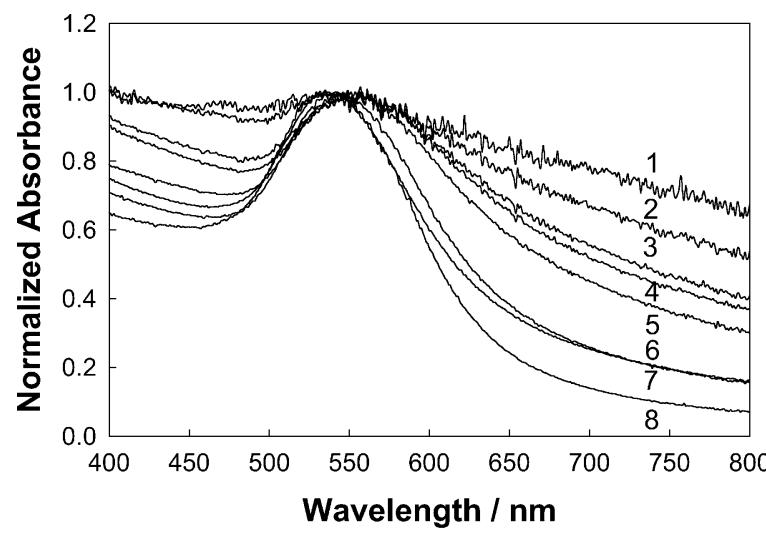

(1) $112 \mu \mathrm{M}$ Con $\mathrm{A}$

(2) $75.0 \mu \mathrm{M}$ Con $\mathrm{A}$

(3) $56.0 \mu \mathrm{M}$ Con $\mathrm{A}$

(4) $37.3 \mu \mathrm{M}$ Con $\mathrm{A}$

(5) $17.4 \mu \mathrm{M}$ Con $\mathrm{A}$

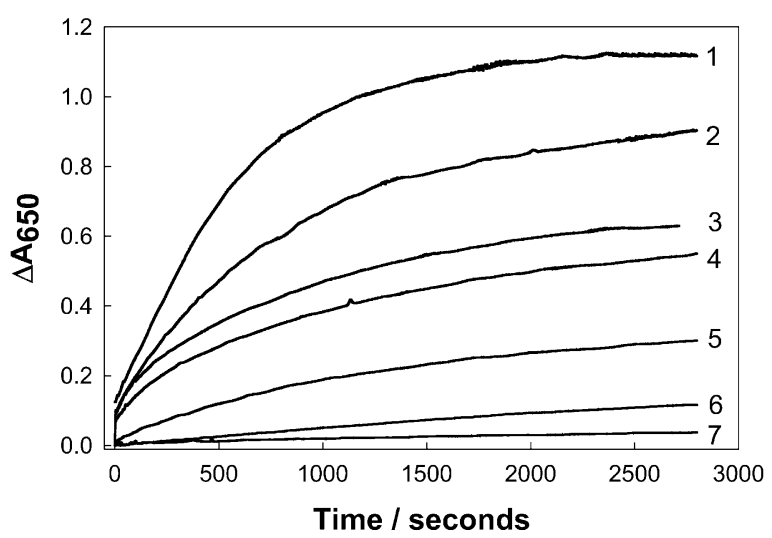

(6) $8.70 \mu \mathrm{M}$ Con $\mathrm{A}$

(7) $4.40 \mu \mathrm{M}$ Con $\mathrm{A}$

(8) $\mathrm{Au} 500 \mathrm{~K}$

Fig. 4. Normalized absorption spectra of $500 \mathrm{~K}$ dextran-coated $20 \mathrm{~nm}$ gold colloids crosslinked
change in absorbance at $650 \mathrm{~nm}$ after the addition of the respective Con A concentrations (bottom) 

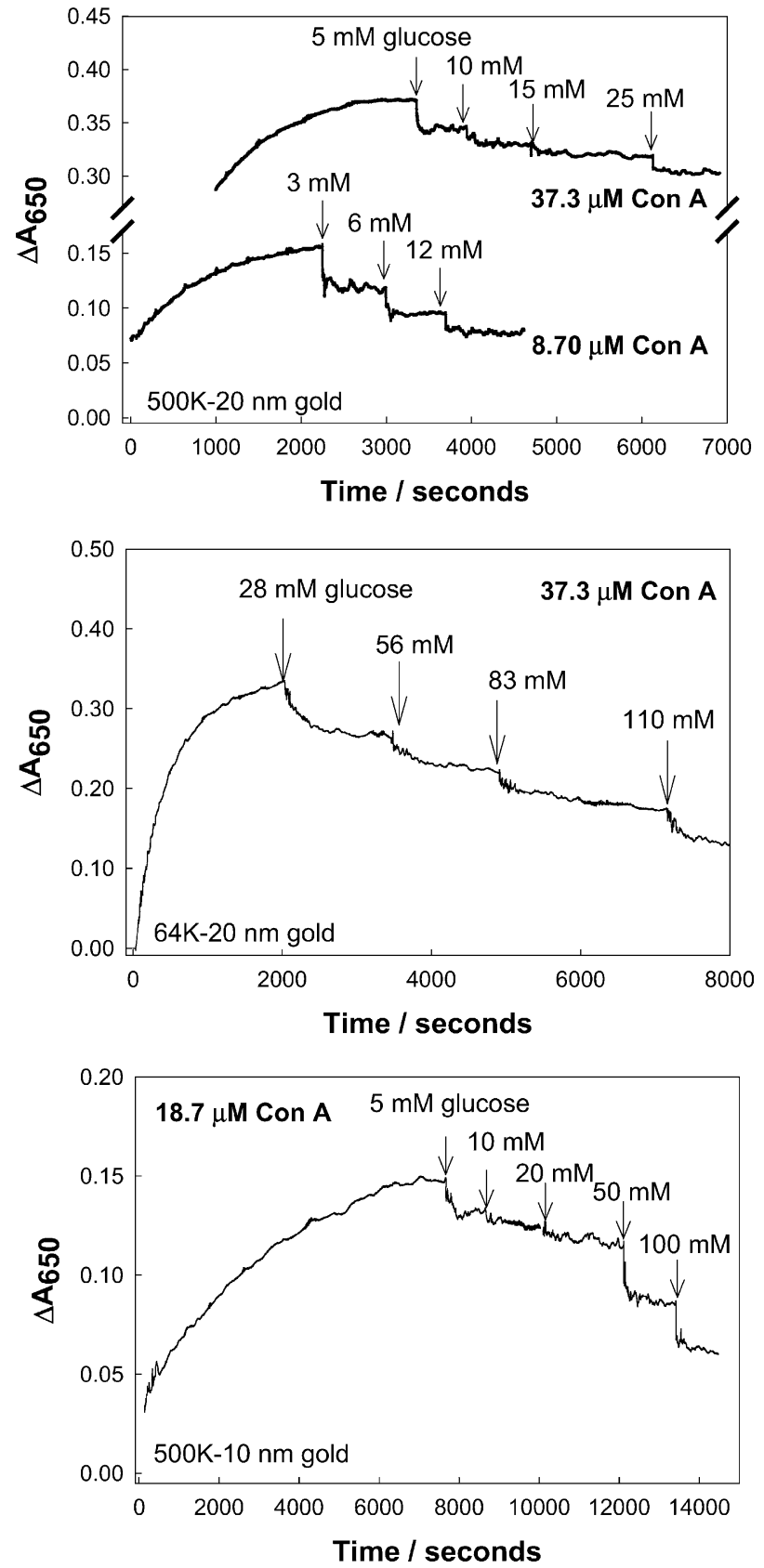

Fig. 5. Change in absorbance at $650 \mathrm{~nm}$ for $500 \mathrm{~K}$ dextran-coated $20 \mathrm{~nm}$ gold colloids with different initial amounts of Con A used to crosslink the system (top), using a 64K dextran-coated gold colloid (middle) and using a $500 \mathrm{~K}$ dextran-coated $10 \mathrm{~nm}$ gold colloid, crosslinked by $18.7 \mu \mathrm{M}$ Con A (bottom).

systems $(<4.4 \mu \mathrm{M}$ Con A) showed a greater response (greater $\Delta A_{650}$ for a smaller glucose concentration), the overall $\Delta A_{650}$ values became too small for practical sensing purposes. Subsequently, $\mathrm{mM}$ glucose concentrations can readily be determined with $500 \mathrm{~K}$ dextran, $20 \mathrm{~nm}$ gold, 4-30 $\mu \mathrm{M}$ Con A aggregates. In all our studies, aggregates within this composition range were stable and did not either self-flocculate or precipitate from solution (data not shown).
We speculated on the role of the dextran coating on the gold colloids, given the presence of its glucose sub-units. Subsequently, we synthesized $64 \mathrm{~K}$ dextran-coated $20 \mathrm{~nm}$ gold nanoparticles. We generally found greater $\Delta A_{650}$ values for these aggregates upon the addition of glucose (Fig. 5, middle), as compared to the $500 \mathrm{~K}$ dextran aggregates, which we have attributed to the reduced total amount of glucose in the system involved in the glucose-Con A-dextran dynamic equilibrium. However, these particles were found to be less stable than their $500 \mathrm{~K}$ dextran counter parts and typically flocculated and precipitated 6-8 $\mathrm{h}$ after preparation.

We investigated the role of reduced gold colloid size on our glucose sensor design (Fig. 5, bottom). We typically found that $500 \mathrm{~K}$ dextran, $10 \mathrm{~nm}$ gold aggregates, typically showed greater $\Delta A_{650}$ values as compared to the $20 \mathrm{~nm}$ aggregates, where the reduced colloid size is thought to reduce the total amount of dextran in the samples, given the same number density of colloids. While this result suggests the possibility of tunable glucose sensing ranges by using different size, equally coated, gold nanoparticles, our experience reveals a greater level of complexity when working with the smaller nanoparticles. One particular difficulty is in their separation and recovery after centrifugation from the reactants.

Finally, it is informative to comment on the reversibility of the sensing system. The nature of the equilibrium and the competitive binding between dextran-Con A and glucose is well known to afford for reversible glucose sensing schemes $[22,23]$. Indeed, many energy transfer and reverse fluorescence resonance energy transfer based sensing schemes have been developed using combinations of donor and acceptor labeled dextran and Con A [25-28], all utilizing the reversibility of the dextran-Con A glucose interaction. In our studies here, it was not possible to remove the glucose from solution to demonstrate the reversibility, where adding water would have simply reduced the net system solution absorbance. However, as we mention below, reversibility of the system can be demonstrated in a polymeric support.

\section{Conclusions}

We have explored the possibility of glucose sensing using the dissociation of Con A-aggregated dextran-coated gold colloids. Our findings reveal that our approach can readily determine $\mathrm{mM}$ changes in solution glucose concentrations in a continuous manner. Moreover, the glucose sensing range of the aggregates can be somewhat tuned depending on the size of the gold colloids employed, the molecular weight of the dextran and the concentration of Con A used to form the sensing aggregate.

The need for tunable glucose sensing ranges has recently been obviated by work from the author's laboratories, which has involved the fabrication of a range of glucose sensing contact lenses for potential use by diabetics [29-31]. Tear glucose levels, which track blood levels, can fluctuate from 
several hundred $\mu \mathrm{M}$ to $\mathrm{mM}$ glucose [29-31]. In addition, all contact lens glucose sensing to date by us has involved optical measurements in the UV-blue, which is plagued by eye autofluorescence. These new aggregate sensors described here have the opportunity to be incorporated into disposable, plastic contact lenses for the non-invasive and continuous monitoring of tear, and therefore blood glucose [29], at wavelengths $>600 \mathrm{~nm}$, alleviating eye autofluorescence, scattered light ( $\lambda^{-4}$ dependence) and over exposure of the eye to UV radiation, as restricted by the US Food and Drug Administration (FDA). In addition, there are no notable interferents in tears for these sensing aggregates. In this regard, it is worth noting that the affinity of the tetravalent protein Con A for glucopyranosides and mannosides has been well studied [32], due its widespread use in glucose sensing [22-28]. While glucose is well known to competitively displace other sugars, such as dextran which only weakly binds [22-28,32], mannosides are also low affinity ligands [32] $\left(K_{\mathrm{d}} \approx 10^{-3} \mathrm{M}\right)$ and galactose does not bind.

\section{Acknowledgements}

This work was supported by the NIH, National Center for Research Resources, RR-08119.

\section{References}

[1] M.R. Robinson, R.P. Eaton, D.M. Haaland, G.W. Koepp, E.V. Thomas, B.R. Stallard, P.L. Robinson, Clin. Chem. 38 (1992) 1618.

[2] H.M. Heise, R. Marbach, T.H. Koschinsky, F.A. Gries, Ann. Occup. Hyg. 18 (1994) 439.

[3] W.F. March, B. Rabinovitch, R. Adams, J.R. Wise, M. Melton, Trans. Am. Soc. Artif. Intern. Organs 28 (1982) 232.

[4] B. Rabinovitch, W.F. March, R.L. Adams, Diabetes Care 5 (1982) 254.
[5] G.M. Schier, R.G. Moses, I.E.T. Gan, S.C. Blair, Diabetes Res. Clin. Pract. 4 (1988) 177.

[6] A. Labande, D. Astrue, Chem. Commun. (2000) 1007.

[7] G. Schmid, Clusters and Colloids from Theory to Applications, VCH, New York, 1994

[8] A. Henglein, J. Phys. Chem. 97 (1993) 5457.

[9] J. Belloni, Curr. Opin. Colloid Interface Sci. 1 (1996) 184.

[10] U. Krreibig, M. Vollmer, Optical Properties of Metal Clusters, Springer, Berlin, 1995.

[11] J.J. Stothoff, A.A. Lazarides, R. Mucic, C.A. Mirkin, R. Letsinger, G.C. Schatz, J. Am. Chem. Soc. 122 (2000) 4640.

[12] A.A. Lazarides, G.C. Schatz, J. Phys. Chem. B 104 (2000) 460.

[13] C.A. Mirkin, J.J. Storhoff, Chem. Rev. 99 (1999) 1849.

[14] R.A. Reynolds, C.A. Mirkinm, R.L. Letsinger, J. Am. Chem. Soc. 122 (2000) 3795.

[15] C.A. Mirkin, R.L. Letsinger, R.L. Mucic, J.J. Storhoff, Nature 382 (1996) 607.

[16] Y. Kim, R.C. Johnson, J.T. Hupp, Nano Lett. 1 (2001) 165.

[17] S.Y. Lin, S.W. Liu, C.M. Lin, C.H. Chen, Anal. Chem. 74 (2002) 330.

[18] N.T.K. Thanh, Z. Rosenzweig, Anal. Chem. 74 (2002) 1624.

[19] K. Aslan, V.H. Pérez-Luna, Langmuir 18 (2002) 6059-6065.

[20] S. Lofas, B. Johnsson, J. Chem. Soc., Chem. Commun. (1990) 1526.

[21] C.S. Weisbecker, M.G. Merritt, G.M. Whitesides, Langmuir 12 (1996) 3763-3772.

[22] R. Ballerstadt, J.S. Schultz, Anal. Chem. 72 (2000) 4185.

[23] A. Yoshizumi, N. Kanayama, Y. Maehara, M. Ide, H. Kitano, Langmuir 15 (1999) 482.

[24] D. Roll, J. Malicka, I. Gryczynski, Z. Gryczynski, J.R. Lakowicz, Anal. Chem. 75 (2003) 3440 .

[25] J.S. Scultz, G. Sims, Biotechnol. Bioeng. Symp. 9 (1979) 65.

[26] J. Shultz, S. Mansouri, I.J. Goldstein, Diabetes Care 5 (1982) 245.

[27] D. Meadows, J.S. Shultz, Talanta 35 (1988) 145.

[28] J.R. Lakowicz, Principles of Fluorescence Spectroscopy, Kluwer/Plenum Press, New York, 1999.

[29] R. Badugu, J.R. Lakowicz, C.D. Geddes, Anal. Chem. 76 (2004) 610.

[30] R. Badugu, J.R. Lakowicz, C.D. Geddes, J. Fluoresc. 13 (2003) 371.

[31] R. Badugu, J.R. Lakowicz, C.D. Geddes, Biophotonics Int. 11 (2004) 50.

[32] J.E. Gestwicki, C.W. Cairo, L.E. Strong, K.A. Oetjen, L.L. Kiessling, J. Am. Chem. Soc. 124 (2002) 14922. 